\title{
On noise processes and limits of performance in biosensors
}

\author{
Arjang Hassibi ${ }^{\mathrm{a})}$ \\ Electrical and Computer Engineering Department, University of Texas at Austin, Austin, Texas 78712 \\ Haris Vikalo and Ali Hajimiri \\ Electrical Engineering Department, California Institute of Technology, Pasadena, California 91125
}

(Received 11 January 2007; accepted 4 May 2007; published online 11 July 2007)

\begin{abstract}
In this paper, we present a comprehensive stochastic model describing the measurement uncertainty, output signal, and limits of detection of affinity-based biosensors. The biochemical events within the biosensor platform are modeled by a Markov stochastic process, describing both the probabilistic mass transfer and the interactions of analytes with the capturing probes. To generalize this model and incorporate the detection process, we add noisy signal transduction and amplification stages to the Markov model. Using this approach, we are able to evaluate not only the output signal and the statistics of its fluctuation but also the noise contributions of each stage within the biosensor platform. Furthermore, we apply our formulations to define the signal-to-noise ratio, noise figure, and detection dynamic range of affinity-based biosensors. Motivated by the platforms encountered in practice, we construct the noise model of a number of widely used systems. The results of this study show that our formulations predict the behavioral characteristics of affinity-based biosensors which indicate the validity of the model. (C) 2007 American Institute of Physics.
\end{abstract}

[DOI: $10.1063 / 1.2748624]$

\section{INTRODUCTION}

Affinity-based biosensors use selective binding and interaction between certain biomolecules (recognition probes) and specific target analytes to determine the presence of the latter in the biological samples. The essential role of the biosensor platform (e.g., microarrays, ${ }^{1,2}$ and immunoassays ${ }^{3}$ ) is to facilitate the binding of the probe-target complexes to produce a detectable signal, which correlates with the presence of the target and conceivably its abundance. The minimal components required for affinity-based detection include a molecular recognition layer (capturing probe) integrated within or intimately associated with a signal-generating physiochemical transducer ${ }^{4}$ and a readout device.

To generate target-specific signal, the target analytes in the sample volume first need to collide with the recognition layer, interact with the probes, and ultimately take part in a transduction process. The analyte motion in typical biosensor settings (e.g., aqueous biological mediums) is dominated by diffusion spreading, which from a microscopic point of view is a probabilistic mass-transfer process (i.e., random-walk events for a single analyte molecule ${ }^{5}$ ). Accordingly, the analyte collisions with the probes become stochastic processes. Moreover, because of the quantum-mechanical nature of chemical bond forming, ${ }^{6-9}$ interactions between the probes and the analyte molecules are also probabilistic, adding more uncertainty to the biosensing procedure. On top of these two processes, we also have the transducer and readout circuitry, which likely adds more noise to the already noisy process.

Beside the inevitable uncertainty associated with the binding, transduction, and readout, in all practical biosensors, binding of other species to the probes (nonspecific

\footnotetext{
${ }^{a)}$ Previously at Electrical Engineering Department, California Institute of Technology, Pasadena, CA 91125; electronic mail: arjang@mail.utexas.edu
}

binding) is also possible. Nonspecific binding (e.g., crosshybridization in DNA microarrays ${ }^{1}$ ) is generally less probable than the specific binding when target analyte and the interfering species have the same abundance. Nonetheless, if the concentration of the nonspecific analyte becomes much higher than the target analyte, nonspecific bindings may dominate the measured signal and hence limit the minimumdetectable level (MDL) of the biosensor platform. ${ }^{10}$ In this paper, we examine all of the aforementioned processes and assess their contributions to the biosensor output signal. Our goal is to introduce a general methodology to model the noise in biosensors, and based on that, evaluate the MDL, highest detection level (HDL), and detection dynamic range (DR).

Initially, in Sec. II, we review the existing stochastic model of the biosensor platforms, ${ }^{11}$ which is essentially a Markov model for the analyte capturing. We proceed by adding to it a probabilistic model for the transduction, probe saturation, and the readout process. In Sec. III, we implement this model to evaluate the uncertainty of the detected signal and the relative noise contributions of all underlying processes as a function of the analyte concentrations, capturing probe density and other characteristics of the biosensor system. Finally, in Sec. IV, demonstrating the practical use of our model, we implement the model to describe the stochastic behavior of impedimetric ${ }^{12}$ and fluorescent-based ${ }^{13}$ biosensors.

\section{BIOSENSOR STATISTICAL MODEL}

\section{A. Analyte motion}

Molecules, cells, and many other analytes immersed in the aqueous mediums of biosensor platforms are subject to thermal fluctuations and, in particular, sensory platforms, 


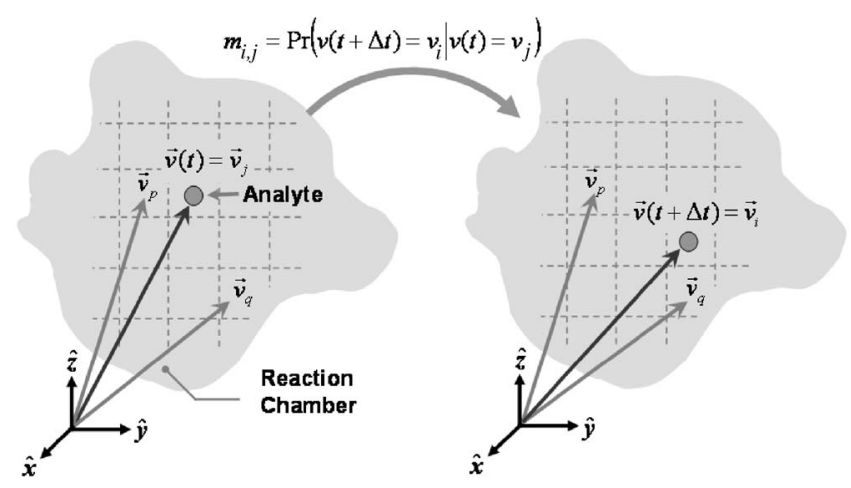

FIG. 1. Markov model for probabilistic motion in the reaction chamber. Each coordinate corresponds to a state of the process into which target analytes can move or from which they can leave. $\mathbf{v}(t)$ represents the location of the analyte particle at time $t$, and $\mathbf{v}_{i}$ defines the coordinates of the state $i$ in the system.

subject to electromagnetic or mechanical forces. While mechanical movement (e.g., convection) and electromagnetic forces (e.g., electrophoresis) are in some sense deterministic, thermal fluctuations are random in nature. Thermal fluctuations of a particle from a microscopic point of view follow the characteristics of a typical random-walk process ${ }^{5}$ (i.e., Brownian motion), which results in a diffusive spreading phenomenon in macroscopic systems.

It is challenging to statistically describe the motion of individual analyte molecules in the general case using the continuity equation formulation. However, we can utilize a discrete-time Markov process ${ }^{14}$ to model the particle stochastic behavior within the reaction chamber. ${ }^{11}$ In this model, we discretize the coordinates of the reaction chamber, where the states of the Markov model correspond to a fixed set of coordinates within the chamber (Fig. 1). The transition probabilities of the Markov process are defined as the probabilities of an analyte moving from one coordinate (state) to another in the given time epoch $\Delta t$. Such a simple model captures the stochastic nature of the process yet is still tractable enough to allow for computation of the probability density function (pdf) of the analyte particles. Additionally, we can easily derive the expected behavior (ensemble average) of the system, a quantity that is generally assessed using a continuity-based equation formulation.

For two arbitrary coordinates $\mathbf{v}_{i}$ and $\mathbf{v}_{j}$ in the system, associated with states $i$ and $j$, the state transition probability from state $i$ to $j$, for an analyte particle in the time interval $\Delta t$, is denoted by $m_{i, j}$,

$$
m_{i, j}=\operatorname{Pr}\left[\mathbf{v}(\Delta t)=\mathbf{v}_{i} \mid \mathbf{v}(0)=\mathbf{v}_{j}\right],
$$

where $\mathbf{v}(0)$ and $\mathbf{v}(\Delta t)$ specify the coordinate of an analyte particle at times 0 and $t$, respectively. The $m_{i, j}$ defined in (1) represents the $(i, j)$ element of the analyte transition matrix $M(\Delta t)\left[M(\Delta t) \in R^{N \times N}\right.$ and $N$ is the total number of states]. If an analyte particle has the probability distribution $x(0)$ at time zero across the state space, where for all $t x(t) \in R^{N}$, then at $\Delta t$,

$$
x(\Delta t)=M(\Delta t) x(0) .
$$

Typical biosensor structures have a large number of analyte particles within the reaction chamber. Provided that their mo- tion is statistically independent, $M(\Delta t)$ and $x(t)$ become independent, resulting in a homogeneous Markov process. Note that this is, in fact, a realistic assumption for most biosensors, because statistical motion in such systems is governed by analyte interaction with the medium molecules and temperature, and not by analyte-analyte interactions. Hence, analytes seldom affect the probabilistic motion of one another. Because of the homogeneity of the Markov process, we are able to calculate the spatial probability distribution of the analyte particles at all time instants that are multiples of $\Delta t$, given the initial distribution $x(0)$ and $M(\Delta t)$, by

$$
x(k \Delta t)=[M(\Delta t)]^{k} x(0),
$$

where $k$ is an integer. If we have $n$ analyte particles in the system we can also define a spatial concentration distribution of the analyte particles $X(t)$, where $X(t)=n x(t)$ and hence

$$
X(t)=n x(k \Delta t)=n[M(\Delta t)]^{k} x(0)=[M(\Delta t)]^{k} X(0),
$$

where $X(0)$ is the initial concentration of the analyte particles. It is important to realize that the matrix $M(\Delta t)$ can theoretically be estimated for any small time increment $\Delta t$, given the exact statistics of the mass transport process described by the continuity equation. ${ }^{11}$

\section{B. Analyte capture}

Now that we have a statistical model of analyte motion within the solution, we need to incorporate the boundary conditions within the biosensor chamber. The boundary conditions for conventional biosensors are either purely reflective (e.g., inert chamber walls and solution surfaces) or selectively absorbing (e.g., surfaces where the capturing probes exist). Incorporation of reflective boundaries into the Markov model is carried out by assigning zero probability for particles to move beyond the boundaries. However, accommodation of selective absorption requires a probabilistic model for the collision and interaction processes.

Analyte collision with the binding sites may be reactive, elastic, or inelastic. ${ }^{15}$ We have previously introduced a probabilistic model to describe the specific binding of an analyte particle $X$ to a single probe $Y$ in an affinity-based biosensor. ${ }^{11}$ To begin with, we assume that the probe $Y$ is confined (immobilized) in a certain coordinate. Now we can argue that any meaningful interaction between $X$ and $Y$ at time $t$ only occurs if $\mathbf{v}(t)=\mathbf{v}_{1}$ (i.e., molecule $X$ is in intimate proximity of $Y$, as shown in Fig. 2). If the bulk-phase reaction between the analytes $X$, and the capturing probes $Y$ has an association rate $k_{1}$, and disassociation rate $k_{-1}$, then we can write

$$
\begin{aligned}
& \stackrel{k_{1}, k_{-1}}{\leftrightarrow} X Y \\
& \frac{d[X Y]}{d t}=k_{1}[X][Y]-k_{-1}[X Y],
\end{aligned}
$$

where the symbol [] indicates concentration of the species. Now based on (5) we can apply the following approximation to find the transition probabilities between the captured state $c$ of the analyte and the collided state $1:^{11}$ 


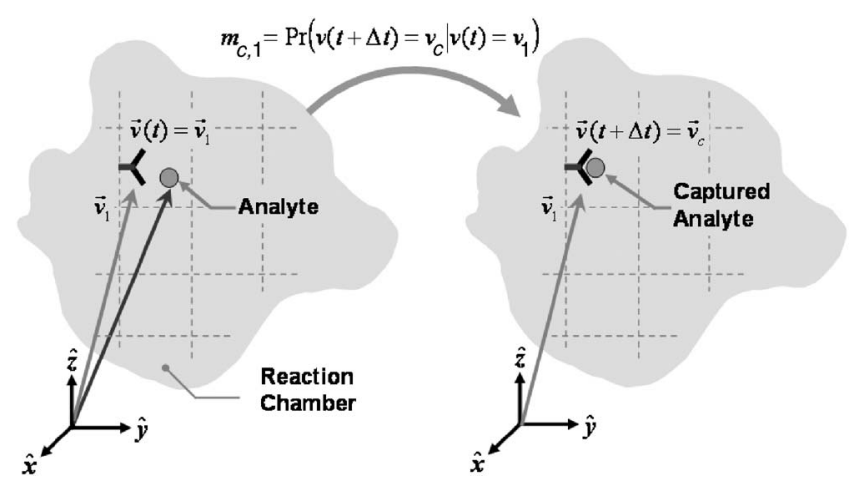

FIG. 2. Markov model for probabilistic capture in the reaction chamber Only when the target analytes get into intimate proximity of the capturing probes (state 1) there is a probability of capturing denote by $m_{c, 1}$.

$$
\begin{aligned}
& m_{1, c}=\alpha k_{1} \Delta t, \\
& m_{c, 1}=k_{-1} \Delta t .
\end{aligned}
$$

The parameters $m_{1, c}$ and $m_{c, 1}$ are also defined as the association and disassociation transition probabilities, respectively. The quantity $\alpha$ basically describes the effects of immobilization of probes and the finite reactive distance between the probe and target. Using (6) for absorbing boundaries and (4) for the mass transfer and reflection cases, we can analyze the binding and motion of the analytes in the biosensor systems in its entirety.

The formulation in (6) assumes infinite capturing capacity for probes, which is not always realistic. If there are $n_{1, Y}$ immobilized capturing sites in state 1 , then the association and disassociation probabilities for the immobilized $Y$ species become functions of $n_{c}(t)$, the number of captured analytes at state $c$, such that

$$
\begin{aligned}
& m_{c, 1}(t)=\alpha k_{1}\left[\frac{n_{1, Y}-n_{c}(t)}{n_{1, Y}}\right] \Delta t, \\
& m_{1, c}=k_{-1} \Delta t,
\end{aligned}
$$

where the term $\left[\left(n_{1, Y}-n_{c}(t)\right)\right] / n_{1, Y}$ is basically the probability of finding a free probe at the capturing site $c$ (see Fig. 3). The incorporation of (7) into (4) clearly results in a nonhomogeneous Markov process, since the transition probabilities have become a function of target analyte distribution, i.e., $M(\Delta t)$ in (4) becomes a function of $n_{c}(t)$. Finding a closed form for the dynamics of such system is challenging; however, we
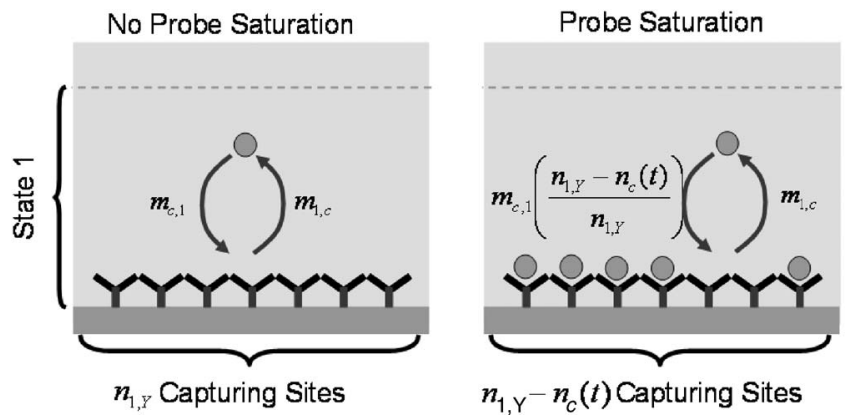

FIG. 3. Modification of association transition probability $m_{c, 1}$, due to probe saturation. will demonstrate in Sec. III how in chemical equilibrium the fluctuations of the system can be quantified.

\section{Transduction}

We define the transduction process and the output signal of the biosensor as a function of $X(t)$, the target concentration distribution within the system. We can represent transduction process by matrix $T$, where $T \in R^{1 \times(N+P)}$ and $P$ being the number of detectable capturing states. Accordingly, the output signal $s(t)$ is defined by

$$
s(t)=T X(t)+u_{T}(t)
$$

where $u_{T}(t)$ is a random process describing the additive noise of the transducer. The matrix $T$ in (8) signifies the process wherein binding results in a detectable signal or parameter. For example, the unit of $T$ in a chemiluminescence-based biosensor ${ }^{16}$ is photons/s target, while in impedimetric biosensors are $\Delta \Omega^{-1} /$ target (see Sec. IV). As implied by (8), the transduction process itself may introduce some uncertainty in the form of an additive noise component which is represented by $u_{T}(t)$. We know from (4) that $X(t)$ is a random process, but if we assume that its expected behavior (ensemble average) is denoted by $\overline{X(t)}$, we can rewrite (8) as

$$
s(t)=T\left[\overline{X(t)}+u_{X}(t)\right]+u_{T}(t)=T \overline{X(t)}+T u_{X}(t)+u_{T}(t) .
$$

In (9), the random process $u_{X}(t)$ essentially describes the deviation of the actual analyte concentration (perturbation) from the expected distribution $\overline{X(t)}$, where $\overline{u_{X}(t)}=0$ for all $t$. The function $T u_{X}(t)$ therefore describes the observed biochemical noise of the system.

Since the transduction mechanism varies tremendously among biosensor platforms, we need to reexamine (9) and derive a formulation which makes their performances comparable. One approach that we propose in this paper is to refer the biosensor output signal to the input of the biosensor system which is essentially the number of analytes in the sample. In this approach the biosensor equivalent signal $s_{b}(t)$ represents the number of perceived target analytes in the sample plus its uncertainty. To carry out the procedure, we divide (9) by the effective gain of the biosensor system which is $T_{\Sigma}=\Delta s(t) / \Delta n=T \overline{X(t)} / n$. The parameter $T_{\Sigma}$ is basically the expected output signal change per analyte concentration change within the system. Now the biosensor master equation expressed in terms of the number of analytes is given by

$$
s_{b}(t)=n+\left(T_{\Sigma}^{-1} T\right) u_{X}(t)+T_{\Sigma}^{-1} u_{T}(t) .
$$

If there are $K$ stages after transducer (see Fig. 4), each with gains $G_{i}$ and additive noise $u_{i}(t)(i=1, \ldots, K)$, we can incorporate them in (10) such that

$$
s_{b}(t)=n+T_{\Sigma}^{-1}\left[T u_{X}(t)+u_{T}(t)+\sum_{i=1}^{K}\left(\prod_{j=1}^{i} G_{j}^{-1}\right) u_{i}(t)\right] \text {. }
$$

Equations (10) and (11) essentially provide the number of observed (or existing) target analytes in the biosensor system. In the next section we apply these stochastic formula- 

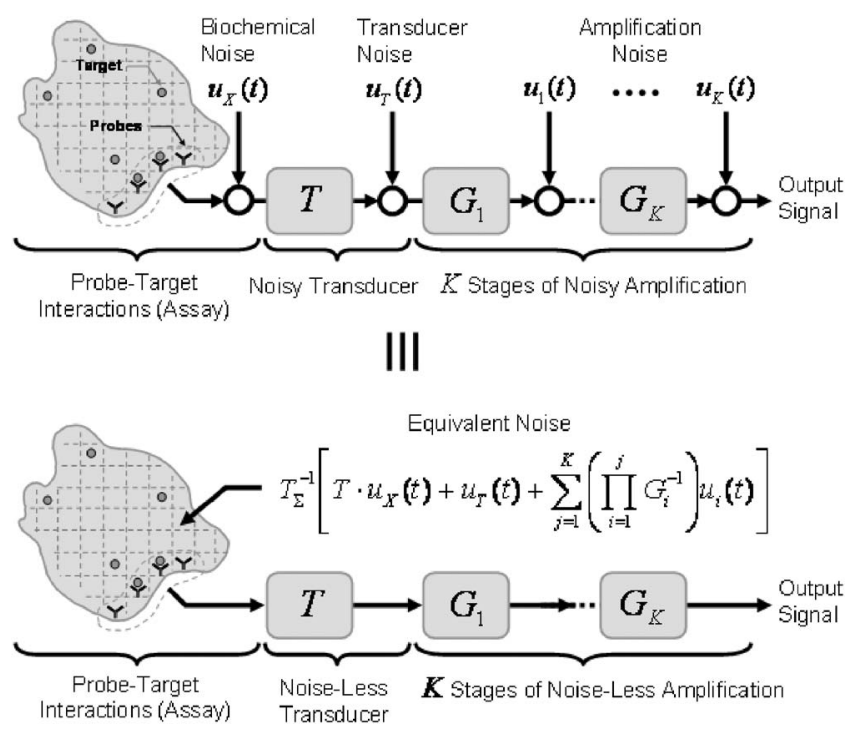

FIG. 4. Block diagram of different stages of a general biosensor platform. Various noise processes originating from different sources may corrupt the signal; however, all of these noise sources can be referred to as the number of analytes in the sample.

tions to calculate the noise characteristics of biosensor platforms.

\section{NOISE AND SIGNAL FLUCTUATION}

At this point we can utilize the transition matrix derived in (4) along with the correct boundary conditions of (7) to simulate various characteristics of the biosensor, and use (9)-(11) to assess the output signal of the system. The simulation of such a system although possible is generally cumbersome, since the number of states is extremely large in practical biosensors. Hence, in the following subsections we will derive the closed form expressions describing the fluctuation of biosensor output. In particular, we will focus on the noise at biochemical equilibrium which, for many practical reasons, is the state where the measurements are preferably carried out.

\section{A. Biochemical fluctuation in equilibrium $\left[T u_{X, E}(t)\right]$}

We can show that when there is no saturation in the biosensor, the general solution for $x(t)$ [and $X(t)$ ] which defines the single analyte probability distribution at time $t$, given an initial condition $x(0)$, is described by the following: ${ }^{11}$

$$
x(t)=\exp \left\{\frac{[M(\Delta t)-I]}{\Delta t} t\right\} x(0)=H(t) x(0),
$$

where $I$ is the identity matrix of the same dimensions as $M(\Delta t)$. Matrix $H(t)$ advances the initial distribution in time and is calculated using the matrix exponential function. It can be shown that $M(\Delta t)$ has a single eigenvalue equal to 1 , and all other eigenvalues of $M(\Delta t)$ have magnitudes less than 1, ensuring a single equilibrium distribution, $x_{E}$ which is the eigenvector associated with an eigenvalue of 1 . Furthermore, it can be shown that $M(\Delta t)$ and $H(t)$ share $x_{E}$, i.e., it holds that

$$
x_{E}=M(\Delta t) x_{E}=H(t) x_{E} .
$$

To quantify the biochemical noise of the system $T u_{X}(t)$, we need to characterize the analyte fluctuation in every state, and particularly in the states for which the transduction occurs (i.e., the states corresponding to nonzero entries of $T$ ). To perform this calculation for the biochemical equilibrium, we first need to evaluate the autocorrelation function of the stationary random processes describing the occupancy of the states denoted by $O_{i, j}(t)$, where $1 \leqslant i \leqslant N$ describes the state of the system and $1 \leqslant j \leqslant n$ represents the target analytes. The occupancy $O_{i, j}(t)$ is essentially an indicator function of the form

$$
O_{i, j}(t)= \begin{cases}1 & \text { if } \mathbf{v}_{j}(t)=\mathbf{v}_{i} \\ 0 & \text { otherwise }\end{cases}
$$

which means that $O_{i, j}(t)=1$ if the $j$ th particle occupies state $i$ at time $t$, and zero otherwise. It can be shown that $R_{i, j}(\tau)$, the autocorrelation of $O_{i, j}(t)$, is identical for all $n$ analytes and can be found using the definition of $H(t)$ to be

$$
R_{i, j}(\tau)=h_{i i}(|\tau|) x_{i, E},
$$

where $x_{i, E}$ is the $i$ th component of $x_{E}$ and $h_{i i}(\tau)$ is the $i$ th diagonal entry of $H(\tau) .{ }^{11}$ Since we have $n$ independent particles in the system, we define the stochastic process $n_{i}(t)$ counting the total number of particles occupying state $i$ as

$$
n_{i}(t)=\sum_{j=1}^{n} O_{i, j}(t)
$$

and hence $X(t)=\left[n_{1}(t) n_{2}(t) \cdots n_{(N+P)}(t)\right]$. Now by implementing (15), we can derive $R_{n_{i}}(\tau)$, the autocorrelation function of $n_{i}(t)$, as

$$
\begin{aligned}
R_{n_{i}}(\tau) & =\overline{\sum_{i=1}^{n} O_{i, j}(t+\tau) \sum_{i=1}^{n} O_{i, j}(t)} \\
& =\left(n^{2}-n\right)\left[\overline{O_{i, j}(t)}\right]^{2}+n R_{i, j}(\tau) \\
& =\left(n^{2}-n\right)\left(x_{i, E}\right)^{2}+n h_{i i}(|\tau|) x_{i, E} .
\end{aligned}
$$

Accordingly, the unilateral (single-sided) power spectral density (PSD) of $n_{i}(t)$ defined by $S_{n_{i}}(f)$ which describes the fluctuations as a function of frequency becomes

$$
S_{n_{i}}(f)=2 \pi\left(n^{2}-n\right)\left(x_{i, E}\right)^{2} \delta(f)+2 n S_{O_{i}}(f),
$$

where

$$
S_{O_{i}}(f)=x_{i, E} \int_{-\infty}^{+\infty} h_{i i}(|\tau|) e^{-j 2 \pi f \tau} d \tau
$$

To find $\sigma_{n_{i}}^{2}$, the variance of $n_{i}(t)$, we have

$$
\sigma_{n_{i}}^{2}=\overline{\sum_{i=1}^{n} O_{i, j}(t) \sum_{i=1}^{n} O_{i, j}(t)}-\left[\overline{\sum_{i=1}^{n} O_{i, j}(t)}\right]^{2}=n x_{i, E}\left(1-x_{i, E}\right) .
$$

It is evident from (4) and (13) that the expected value of $n$ independent particles in state $i$ becomes $n x_{i, E}$. Thus, $\overline{X_{E}}$ the expected concentration at equilibrium becomes 


$$
\overline{X_{E}}=\lim _{t \rightarrow \infty} \overline{X(t)}=\lim _{t \rightarrow \infty}\left[n_{1}(t) n_{2}(t) \cdots n_{(N+P)}(t)\right]^{T}=n x_{E} .
$$

Based on the definition of $u_{X}(t)$, now for the equilibrium fluctuation $u_{X, E}(t)=\lim _{t \rightarrow \infty} u_{X}(t)$ we can derive the expected value $\overline{u_{X, E}(t)}$, and the variance $\sigma_{X}^{2}$, where

$$
\begin{aligned}
\overline{u_{X, E}(t)} & =0, \\
\sigma_{X}^{2}= & n\left[x_{1, E}\left(1-x_{1, E}\right) x_{2, E}\left(1-x_{2, E}\right) \cdots x_{(N+P), E}\right. \\
& \left.\times\left(1-x_{(N+P), E}\right)\right]^{T} .
\end{aligned}
$$

Now by using (22), we easily compute for the expected value of the output noise fluctuation $\overline{T u_{X, E}(t)}$ and its variance $\sigma_{S}^{2}$ at equilibrium

$$
\begin{aligned}
& \overline{T u_{X, E}(t)}=0, \\
& \sigma_{S}^{2}=T^{(2)} \sigma_{X}^{2},
\end{aligned}
$$

where $T^{(2)}=\left[\begin{array}{llll}t_{1}^{2} & t_{2}^{2} & \cdots & t_{(N+P)}^{2}\end{array}\right]$ is the element-by-element square of the transduction matrix $T=\left\lfloor t_{1} t_{2} \cdots t_{(N+P)}\right\rfloor$. It is important to realize that $\sigma_{S}^{2}$ is the variance of the unavoidable noise of the system, which is basically the biochemical shot noise originating from the quantum nature of binding events. This particular noise is inherent to affinity-based biosensors and the uncertainty (and certainty) limitation which it establishes is often referred to as the quantum-limit (QL) detection level. ${ }^{17,18}$

Note that while (13)-(23) are derived assuming a homogeneous process (i.e., no probe saturation and no analyteanalyte molecular interactions), the same methodology can be adopted for nonhomogeneous processes, given that an equilibrium distribution exists for the system. The only necessary modification is that a transition matrix at the equilibrium for nonhomogeneous process must be derived to calculate $h_{i i}(|\tau|)$. Essentially in this approach we perform linearization, and the method is thus valid when the fluctuations of the concentration are small compared to the ensemble average concentration (i.e., small perturbation).

\section{B. Output signal fluctuation at equilibrium $\left[T u_{X, E}(t)+u_{T}(t)\right]$}

Although the biochemical noise can easily be quantified by the capturing probability and vector $x_{E}$, presenting a gen- eral stochastic model for the transducer noise $u_{T}(t)$ appears to be more difficult. The main reason is that the additive noise of the transduction process is platform dependent and may originate from fundamentally different physical processes for different transducers. Nevertheless, we can still evaluate the characteristics of the system for a number of specific cases.

The simplest case is when $u_{T}(t)$ is an additive signal independent of the binding process, with expected value of zero and variance of $\sigma_{T}^{2}$. In this case the observed output noise of the biosensor becomes $T u_{X, E}(t)+u_{T}(t)$ with the following expected value $\overline{T u_{X, E}(t)+u_{T}(t)}$ and variance $\sigma_{S}^{2}$ :

$$
\begin{aligned}
& T u_{X, E}(t)+u_{T}(t)=0, \\
& \sigma_{s}^{2}=T^{(2)} \sigma_{X}^{2}+\sigma_{T}^{2} .
\end{aligned}
$$

Now that we have the fluctuation characteristics of the biochemical noise at equilibrium, we can revisit the biosensor master equation. Based on (21), we have $T_{\Sigma}=T \overline{X(t)} / n=T x_{E}$. Therefore, the master equation at equilibrium, constraining our considerations to only biochemical noise and additive transducer noise, becomes

$$
\begin{aligned}
s_{b, E}(t) & =\lim _{t \rightarrow \infty} s_{b}(t)=n+T_{\Sigma}^{-1}\left[T u_{E, X}(t)+u_{T}(t)\right] \\
& =n+\frac{T u_{X, E}(t)+u_{T}(t)}{T x_{E}} .
\end{aligned}
$$

\section{Signal-to-noise ratio and noise figure definitions}

We define the signal-to-noise ratio (SNR) of a biosensor platform as the ratio of the square of the detected target analyte (i.e., signal power) over its noise variance. Using (25), we can write the general expression for the SNR as

$$
\mathrm{SNR}=\frac{\left[\overline{s_{b, E}(t)}\right]^{2}}{\overline{\left[s_{b, E}(t)-s_{b, E}(t)\right]^{2}}} .
$$

By employing (24) and (25), we can rewrite (26) as

$$
\mathrm{SNR}=\frac{n^{2}}{T_{\Sigma}^{2}\left[T^{(2)} \bar{\sigma}_{X}^{2}+\bar{\sigma}_{T}^{2}\right]}=\frac{\left(n T_{\Sigma}\right)^{2}}{T^{(2)} \bar{\sigma}_{X}^{2}+\bar{\sigma}_{T}^{2}},
$$

or

$$
\mathrm{SNR}=n \frac{T_{\Sigma}^{2}}{\left\{t_{1}^{2} x_{1, E}\left(1-x_{1, E}\right)+t_{2}^{2} x_{2, E}\left(1-x_{2, E}\right)+\cdots+t_{(N+P)}^{2} x_{(N+P), E}\left(1-x_{(N+P), E}\right)\right\}+\sigma_{T}^{2} / n} .
$$

The first term in the denominator of (26) and (27) describes the biochemical shot noise (Poisson noise) of the biosensor, while the second term is the added noise of the transducer . It is again important to emphasize that the biochemical shot noise is unavoidable and essentially inherent to the system. Hence, the SNR of the biosensor excluding any transducer noise (i.e., ideal detector) has an upper bound defined by this shot noise which is generally referred to as the QL SNR. 
We can further generalize (27) for the case where the biosensor comprises of other amplification stages as in (11). In this case, the SNR becomes

$$
\mathrm{SNR}=\frac{\left(n T_{\Sigma}\right)^{2}}{T^{(2)} \sigma_{X}^{2}+\sigma_{T}^{2}+\sum_{i=1}^{K}\left(\prod_{j=1}^{i} G_{j}^{-1}\right) \sigma_{i}^{2}},
$$

where $\sigma_{i}^{2}$ represents the variance of the noise added by the $i$ th amplification stage.
In addition to SNR, we can define the noise figure (NF) of the biosensor as another figure of merit for the sensing system. This figure of merit essentially describes the ratio of the added noise to the input noise which is the unavoidable shot noise. We define NF as

$$
\mathrm{NF} \equiv \frac{\text { total output noise power }}{\text { output noise power due to input source }},
$$

and by applying (23)-(25) and for simplicity referring everything to the transducer output we have

$$
\begin{aligned}
\mathrm{NF} & =1+\frac{\sigma_{T}^{2}}{T^{(2)} \sigma_{X}^{2}}+\frac{\sum_{i=1}^{K}\left(\prod_{j=1}^{i} G_{j}^{-1}\right) \sigma_{i}^{2}}{T^{(2)} \sigma_{X}^{2}} \\
& =1+\left(\frac{1}{n}\right) \frac{\sigma_{T}^{2}+\sum_{i=1}^{K}\left(\prod_{j=1}^{i} G_{j}^{-1}\right) \sigma_{i}^{2}}{t_{1}^{2} x_{1, E}\left(1-x_{1, E}\right)+t_{2}^{2} x_{2, E}\left(1-x_{2, E}\right)+\cdots+t_{(N+P)}^{2} x_{(N+P), E}\left(1-x_{(N+P), E}\right)} .
\end{aligned}
$$

Equations (28) and (31) both indicate that by increasing the concentration (or quantity) of the analytes, the system generates more noise although SNR increases and NF decreases. This phenomenon, which one expects from the inherent shot noise of binding events, sets no upper bound for the concentration levels that the biosensor is able to detect. However, from (7) we know that this is not practically justifiable since the probe saturation will eventually occur as we keep increasing the amount of analytes. In the next subsection, we will examine saturation to assess the upper bound of the detection and evaluate the dynamic range of the biosensor.

\section{Saturation and dynamic range}

To find the distribution of analyte particles in the case where the number of capturing probes is finite, we need to incorporate (7) into (12) and compute the equilibrium concentration from (13). This basically results in finding the equilibrium state of a nonhomogeneous Markov process, which is numerically possible but difficult. One alternative approach is to use the simplifying assumption that the analyte molecules in the solution are not depleted by the binding events (i.e., only a very small fraction of analytes is captured by the probes). Although this limits the generality of the solution, it is still applicable to most biosensor platforms. In this case, for each capturing site in state 1 in our model described in Sec. II A, we write the following balance equation at the equilibrium:

$$
n_{c, S} m_{1, c}=n m_{c, 1}\left(\frac{n_{1, Y}-n_{c, S}}{n_{1, Y}}\right),
$$

where $m_{c, 1}, m_{1, c}$ are the transition probabilities from (6) and $n_{c, S}$ is the number of the captured particles when the system is in saturation. Solving (32) to find $n_{c, S}$, we have

$$
n_{c, S}=\frac{n\left(m_{c, 1} / m_{1, c}\right)}{1+\left(n / n_{1, Y}\right)\left(m_{c, 1} / m_{12, c}\right)} .
$$

Note that the parameter $m_{c, 1} / m_{1, c}$ is basically equivalent to $n_{c}(t) / n=x_{c, E}$, which is the analyte capturing probability without saturation. Hence, we use (33) to find $x_{c, S}$, the capturing probability with saturation, as a function of $x_{c, E}$ and $n_{1, Y}$,

$$
x_{c, S}=\left(\frac{n_{c, S}}{n}\right)=\frac{\left(m_{c, 1} / m_{1, c}\right)}{1+\left(n / n_{1, Y}\right)\left(m_{c, 1} / m_{1, c}\right)}=\frac{x_{c, E}}{1+\left(n / n_{1, Y}\right) x_{c, E}} .
$$

The value of $x_{c, S}$ in (34) is basically an entry of $x_{S}$, the equilibrium distribution vector of the system in the presence of saturation. The limits of $x_{S}$ and $x_{c, S}$ when $n \rightarrow 0$ are $x_{E}$ and $x_{c, E}$, respectively, which are the nonsaturated equilibrium distribution and capturing probability. The other limit of $x_{S}$ is zero, obtained by letting $n \rightarrow \infty$. It is important to realize that the value of $n_{1, Y}$ in the general case is different for different states of the system and can be represented by vector $n_{Y}$ $\in R^{(N+P)}$ which has finite entries corresponding to the states with capturing probes, and infinite otherwise.

The most important impact of saturation on our formulations is the breakdown of the linearity assumption between the input (analyte concentration) and the biosensor output signal which enabled the calculation of the SNR and NF in the previous section. Equation (33) basically describes a nonlinear and monotonic relationship between the biosensor input and output. Because of the monotonicity, we can argue that the input can always be evaluated by observing the output of the system, but the concern for the validity of SNR expression is caused by observing the effect of saturation 
(nonlinearity) on the detection uncertainty. In order to evaluate this effect, we use the same approach that we had in evaluating SNR of a linear system, which is referring the output signal plus its noise to the input. The difference here is their nonlinear relationship, which alters the function $T_{\Sigma}$.

First we derive the output fluctuation of the biosensor system in the presence of saturation. Equation (22) indicates that the fluctuation of the number of captured analytes is a function of the capturing probability and $n$. This is independent of the relationship between $n$ and $n_{c, S}$. Thus, we can derive the expected value and variance of the fluctuation of the captured analytes in saturation as

$$
\begin{aligned}
\overline{u_{X, E}(t)} & =0, \\
\sigma_{X}^{2}=n & {\left[\frac{x_{1, E}}{1+\left(n x_{1, E} / n_{1, Y}\right)}\right.} \\
& \times\left(1-\frac{x_{1, E}}{1+\left(n x_{1, E} / n_{2, Y}\right)}\right) \cdots \frac{x_{(N+P), E}}{1+\left(n x_{(N+P), E} / n_{(N+P), Y}\right)} \\
& \left.\times\left(1-\frac{x_{(N+P), E}}{1+\left(n x_{(N+P), E} / n_{(N+P), Y}\right)}\right)\right]^{T},
\end{aligned}
$$

and we can use (35) for the biosensor output fluctuation in (24).

In saturation, the parameter $T_{\Sigma}$ which we defined as $\Delta s(t) / \Delta n$ is derived by calculating the derivative of (33) with respect to $n$ for all states where the transduction occurs. Using the derivative of (34) we have

$$
\Delta n_{c, S}=\frac{x_{c, E}}{\left[1+\left(n x_{c, E} / n_{1, Y}\right)\right]^{2}} \Delta n,
$$

and therefore, by considering the transduction process,

$$
\begin{aligned}
\Delta s(t)= & T\left[\frac{x_{1, E}}{\left[1+\left(n x_{1, E} / n_{1, Y}\right)\right]^{2}} \frac{x_{2, E}}{\left[1+\left(n x_{2, E} / n_{2, Y}\right)\right]^{2}} \cdots\right. \\
& \left.\frac{x_{(N+P), E}}{\left[1+\left(n x_{(N+P), E} / n_{(N+P), Y}\right)\right]^{2}}\right]^{T} \Delta n=T_{\Sigma} \Delta n,
\end{aligned}
$$

which essentially denotes the value of $T_{\Sigma}$ in saturation.

Now that we calculated both $T_{\Sigma}$ and $\sigma_{X}^{2}$ in (35) and (37), we can utilize them in (27) and (31) to find the SNR and NF of the biosensor in saturation. Based on (27), the SNR of a biosensor system without saturation should increase when $n$ increases. However, in saturation, according to the results of (35) and (37), we observe that $T_{\Sigma}$ becomes proportional to $1 / n^{2}$ when $n$ is large, while $\sigma_{X}^{2}$ becomes a constant. Hence, the SNR of the system becomes proportional to $1 / n^{2}$ as $n$ becomes large, which suggest an increase in the uncertainty (noise) of the input referred signal when the biosensor saturates. This is an extremely important outcome of our formulations, since it establishes the fact that the biosensor SNR is not a monotonic function but fundamentally a concave function where SNR $\rightarrow 0$ in both limits of the input (i.e., $n \rightarrow 0$ and $n \rightarrow \infty)$.

To evaluate the range of detection of biosensors, similar to all sensing systems, we are required to find the MDL as well as the HDL. On the lower side of sensing, we define the
MDL or $n_{\mathrm{MDL}}$ as the analyte level where the biosensor SNR is equal to some minimum acceptable value $\mathrm{SNR}_{\min }$. However, based on the results of (35)-(37), for the same $\mathrm{SNR}_{\min }$, we will have another analyte level which is larger than $n_{\mathrm{MDL}}$. This value is basically HDL and the analyte concentration level associated with it is $n_{\mathrm{HDL}}$. Consequently, the dynamic range of the biosensor is defined as

$$
\mathrm{DR}=\frac{n_{\mathrm{HDL}}}{n_{\mathrm{MDL}}} .
$$

The closed form for DR is generally complicated and is derived by solving (27) for $\mathrm{SNR}_{\min }$. As we will show in Sec. IV, the value of DR for biosensors can easily be evaluated numerically for any given value of $\mathrm{SNR}_{\min }$.

\section{E. Effects of interference, non-specific binding, and background}

The expressions for noise, SNR, and DR in the previous subsections all assumed that there is only one analyte species (the target) that can bind to the capturing probes. However, in all practical biosensors, other species coexist with the target in the sample, and they may or may not bind to the probes. We call such species interferers, and their capturing incidents nonspecific bindings. Accordingly, the signal generated by the transducer which originates from nonspecific bindings is called nonspecific signal, or in certain biological detection platforms it is referred to as the background signal.

A nonspecific binding event (e.g., cross-hybridization in DNA microarrays ${ }^{1}$ ) is generally less probable than the specific binding when target analyte and the interfering species have the same abundance in the sample. Nevertheless, when the ratio of the target analyte to the nonspecific analyte is small, contributions of nonspecific binding may dominate the measured signal. To incorporate the effects of nonspecific binding in the previously discussed formulations, we first need to assess the uncertainty associated with the concentration of interferers. This basically defines whether we should consider nonspecific binding incidents coming from a deterministic or a random concentration.

To examine the general case of detection in the presence of interference, we assume that $m$ interferers coexist with the target in the sample, and denote their amounts by $n^{(1)}, n^{(2)}, \ldots$, and $n^{(m)}$, where for the $i$ th specie the expected number of analytes is $\overline{n^{(i)}}$, with variance of $\sigma_{(i)}^{2}$. Obviously, when the interferer concentration is known (deterministic interference), we will have $\overline{n^{(i)}}=n^{(i)}$ and $\sigma_{(i)}^{2}=0$. Now in the general case we can rewrite (25), the equilibrium master equation with interference, as

$$
\begin{aligned}
s_{b, E}(t)= & +T_{\Sigma}^{-1}\left\{T \sum_{i=1}^{m} \overline{n^{(i)}} x_{E}^{(i)}\right. \\
& \left.+T\left[u_{X, E}(t)+\sum_{i=1}^{m} u_{X, E}^{(i)}(t)\right]+u_{T}(t)\right\},
\end{aligned}
$$

where $x_{E}^{(i)}$ and $T u_{X, E}^{(i)}(t)$ are the equilibrium distribution and biochemical noise of the $i$ th interferer specie, respectively. It 
TABLE I. Signal-to-noise ratio (SNR) and noise figure (NF) of biosensor systems when (a) only the target analyte is in the system, (b) the target analyte and the $m$ interfering species coexist in the system, and (c) when the system in addition has $K$ noisy amplification stages.

\begin{tabular}{ccc}
\hline \hline & Signal-to-noise ratio (SNR) & Noise figure (NF) \\
\hline a & $\frac{n^{2} T_{\Sigma}^{2}}{T^{(2)} \sigma_{X}^{2}+\sigma_{T}^{2}}$ & $1+\frac{\sigma_{T}^{2}}{T^{(2)} \sigma_{X}^{2}}$ \\
b & $\frac{n^{2} T_{\Sigma}^{2}}{T^{(2)} \sigma_{X}^{2}+T^{(2)} \sum_{i=1}^{m} \sigma_{X(i)}^{2}+\sigma_{T}^{2}}$ & $1+\frac{\sigma_{T}^{2}}{T^{(2)} \sigma_{X}^{2}}+\frac{\sum_{i=1}^{m} \sigma_{X(i)}^{2}}{\sigma_{X}^{2}}$ \\
$\mathrm{c}$ & $\frac{n^{2} T_{\Sigma}^{2}}{T^{(2)} \sigma_{X}^{2}+T^{(2)} \sum_{i=1}^{m} \sigma_{X(i)}^{2}+\sigma_{T}^{2}+\sum_{i=1}^{K}\left(\Pi_{j=1}^{i} G_{j}^{-1}\right) \sigma_{i}^{2}}$ & $1+\frac{\sigma_{T}^{2}}{T^{(2)} \sigma_{X}^{2}}+\frac{\sum_{i=1}^{m} \sigma_{X(i)}^{2}}{\sigma_{X}^{2}}+\frac{\sum_{i=1}^{K}\left(\Pi_{j=1}^{i} G_{j}^{-1}\right) \sigma_{i}^{2}}{T^{(2)} \sigma_{X}^{2}}$ \\
\hline \hline
\end{tabular}

is straightforward to show that $\sigma_{X(i)}^{2}$, the variance of $u_{X, E}^{(i)}(t)$, can be calculated as

$$
\sigma_{X(i)}^{2}=\overline{n^{(i)}}\left[\sigma_{(i)}^{2} A^{(i)}+B^{(i)}\right]^{T},
$$

where

$$
A^{(i)}=\left[\left(x_{1, E}^{(i)}\right)^{2}\left(x_{2, E}^{(i)}\right)^{2} \cdots\left(x_{(N+P), E}^{(i)}\right)^{2}\right],
$$

and

$B^{(i)}=\left[x_{1, E}^{(i)}\left(1-x_{1, E}^{(i)}\right) x_{2, E}^{(i)}\left(1-x_{2, E}^{(i)}\right) \cdots x_{(N+P), E}^{(i)}\left(1-x_{(N+P), E}^{(i)}\right)\right]$.

By employing (40), we can repeat the same procedure as described in Sec. III C to find the SNR and NF of the system, given that the values of all $\overline{n^{(i)}}$ and $\sigma_{(i)}^{2}$ are known. In Table I, we have listed the formulation for SNR and NF with interference described by (38).

To find the DR of the system with interference, we need to find $n_{\min }$ and $n_{\max }$ in the presence of interference. Finding $n_{\min }$ requires solving the modified version of (29), as shown in Table I. To derive $n_{\max }$, we should do the same thing but solve the balance equation (32) in the presence of interferers, which can be very complicated. It is important to realize that in most biosensors the concentration levels of analytes exposed to capturing probes are kept low enough to ensure that

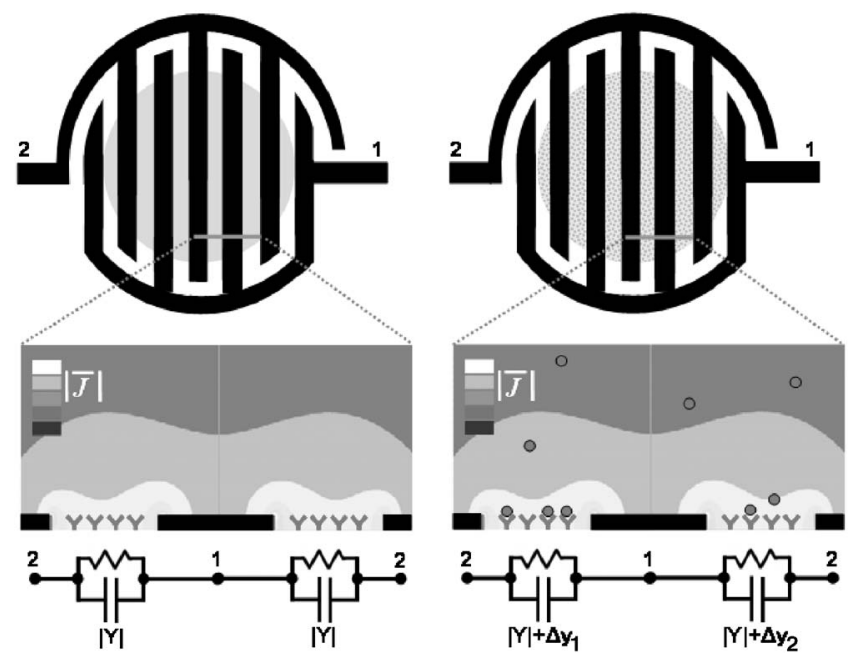

FIG. 5. Impedimetric biosensor where binding of analytes alters the impedance $Z$ (or admittance $Y$ ) between the fingers of an interdigitated electrode structure. The change in overall impedance corresponds to the amount of captured analytes. the background and nonspecific binding do not drive the system into saturation. As a result, the upper limit of detection $n_{\max }$ can still be dominated by the captured target analyte rather than the interferers. Thus, to find $n_{\max }$ in biosensors we generally do not need to take into account the interferer analytes, which makes the calculation less complicated.

\section{MODEL IMPLEMENTATION}

To implement our model, in this section, we look at two different biosensor platforms. The systems under analysis have different transducers (impedimetric and fluorescence based); however, we will demonstrate that for both we can use the described modeling method to evaluate and compare their limits of detection.

\section{A. Impedimetric biosensor}

In impedimetric biosensors the detection relies on the impedance of an electrode-electrolyte interface which can monotonically increase (or decrease) by analyte bindings. In some arrangement, the intrinsic characteristics of the analytes (e.g., charge) are used to the capacitance (and accordingly the impedance) of the electrode-electrolyte interface, ${ }^{13,19-22}$ while in other arrangements, electroactive labels are used ${ }^{23-25}$ to enhance the impedance changes.

In Fig. 5, we have illustrated an example of a microfabricated impedimetric biosensor where an interdigitated electrode-electrolyte structure is used as the transducer. The capturing probes are immobilized between the fingers of the interdigitated structure and binding of analytes changes the conductivity of the solution near the surface by changing the mobility of the free ions. This subsequently modifies the impedance of the electrode-electrolyte transducer system (i.e., ports 1 and 2 in Fig. 5), making the impedance an indication of the target analyte concentration.

In Fig. 6, we have shown the detection circuitry of this impedimetric biosensor which consist of an excitation volt-

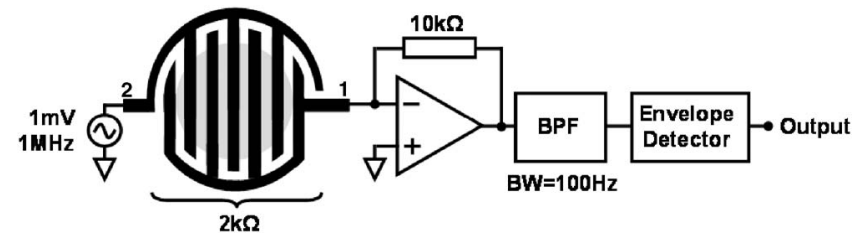

FIG. 6. Detection circuitry for the impedimetric biosensor. 
TABLE II. Characteristics of the impedimetric biosensor.

\begin{tabular}{|c|c|}
\hline Capturing probability & $x_{E}=\left[\begin{array}{llllll}0.3 & 0.45 & 0.6 & 0.6 & 0.45 & 0.3\end{array}\right] \times 10^{-5}$ \\
\hline Probe density & $n_{Y}=\left[\begin{array}{llllll}2 & 5 & 7 & 7 & 5 & 2\end{array}\right] \times 10^{5}$ \\
\hline Reaction chamber volume & $V=10 \mu 1$ \\
\hline Admittance transfer matrix & $\begin{array}{l}\Delta Y=\left[\begin{array}{llllll}2 & 1.5 & 1.2 & 1.2 & 1.5 & 2\end{array}\right] \times 10^{-7} \Omega^{-1} / \\
\text { target }\end{array}$ \\
\hline Transduction matrix & $T=\left[\begin{array}{lllllll}2 & 1.5 & 1.2 & 1.2 & 1.5 & 2\end{array}\right] \times \mu \mathrm{V} /$ target \\
\hline Transducer noise & $\sigma_{T}=\sqrt{k T\left(2 \times 10^{4}\right) B W} \approx 180 \mathrm{nV}$ \\
\hline Interferer capturing probability & $x_{E}^{(1)}=\left[\begin{array}{lllllll}0.3 & 0.45 & 0.6 & 0.6 & 0.45 & 0.3\end{array}\right] \times 10^{-8}$ \\
\hline Interferer variation & $\sigma_{(1)}^{2}=0$ \\
\hline
\end{tabular}

age source at $1 \mathrm{MHz}$, transimpedance amplifier (TIA), bandpass filter, and an envelope detector. Any change in impedance of the transducer changes the current going through structure and subsequently the output of the amplifier which is finally detected by the ideal envelope detector. In Table II, we list the characteristics of this biosensor which is required to calculate the limits of performance of this system. The capturing probabilities are a function of target-probe interac-
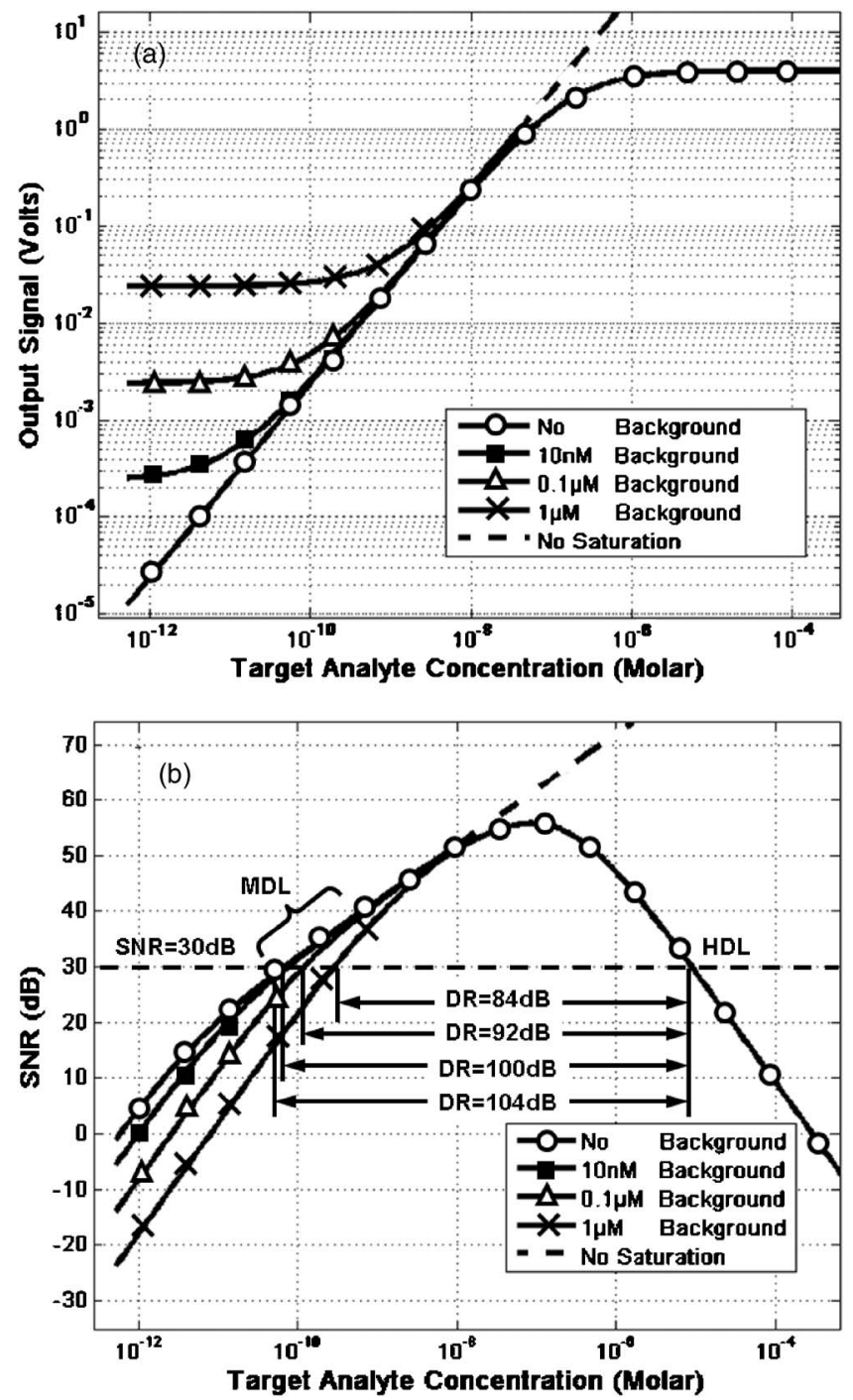

FIG. 7. Performance result of the impedimetric biosensor. In (a) the biosensor output signal is plotted vs the target analyte concentration. In (b) the SNR of the system is plotted and the DR is calculated for the $\mathrm{SNR}_{\text {min }}$ of $30 \mathrm{~dB}$. tion and also the capturing area. These values might seem quite small; however, they are a good approximate for typical biosensor systems where the surface to volume ratio is small. In Table II, we have also listed the transduction matrix, probe density, and the transducer (TIA) additive noise. It is important to realize that the entries of the transduction matrix basically correspond to the different capturing areas of the transducer structure which have different numbers of capturing probes and capturing probability.

In Fig. 7, based on our modeling method we have illustrated the performance of this impedimetric biosensor. In Fig. 7(a), we show the output of the biosensor as a function target analyte concentration. As expected the output signal of this system initially increases linearly with the analyte concentration level but it plateaus eventually due to the saturation. The probabilistic model not only predicts this behavior but also quantifies the background signal of the system which originates from the nonspecific bindings. In Fig. 7(b), we show the SNR of this biosensor based on (26)-(29). Without saturation we expect the SNR to increase monotonically, our formulations quantify how SNR decreases as the analyte concentration level increases. One important observation here is the fact that SNR is more sensitive to interference at low concentration levels, an important empirical observation which has been previously reported in different biosensor systems. ${ }^{26}$ The HDL of this system is limited by saturation and finite number of capturing probe density. However, our results show that the MDL is interference dependent. The overall $\mathrm{DR}$ of this system for $\mathrm{SNR}_{\min }=30 \mathrm{~dB}$ varies between 84 and $104 \mathrm{~dB}$ depending on the interference concentration. The noise of the transducer and the detection circuitry has little effect on the overall performance of the system which indicates that the biochemical noise and probe saturation are performance-limiting factors in this impedimetric biosensor.

\section{B. Fluorescent-based biosensor}

In this example we implement our model for a fluorescent-based biosensor system. We have chosen this particular biosensor system for two main reasons. One is the generality of this system which is currently a widely used detection method in different assays. The second reason is to illustrate the versatility of our modeling method which can unify the performance metrics of different biosensors

The example biosensor platform is illustrated in Fig. 8. In this system, the capturing probes are immobilized at the bottom of the microtiter plate and the analytes are all fluorescent labeled. After an incubation phase, the solution containing analytes is removed from the well and the captured analytes (specific and nonspecific) are quantified using a fluorescence intensity detector. The detector in this example consists of an excitation light source which uniformly excites the captured fluorescent labels, an emission filter which blocks the excitation source while passing the emitted light of the labels, and a photodetector. The photodetector consists of a photodiode and a charge-integrating TIA (CTIA). In Table III we have listed the specification of this system including the transduction gain and signal-dependent noise 

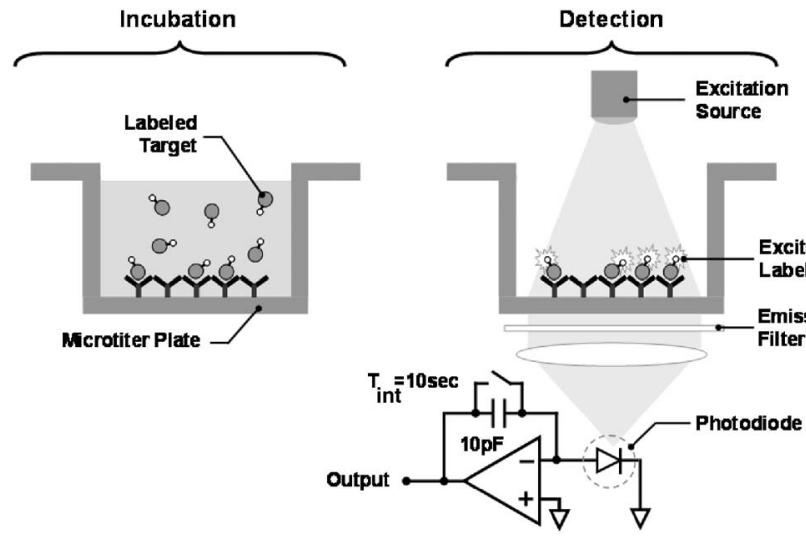

FIG. 8. Fluorescence-based biosensor where binding of labeled analytes is detected after the incubation phase. The emitted light from the fluorescent labels is detected by the photodiode connected to a charge integrating transimpedance amplifier (CTIA).

variance of the transducer. It is important to realize that in this system we have intentionally selected the assay specifications similar to the impedimetric biosensor example, although the transduction mechanism and detector are totally different.

In Fig. 9, we have the performance results of this biosensor. The output of the system as a function of analyte concentration [see Fig. 9(a)] is similar to the impedimetric biosensor; however, the voltage level is lower because of the lower transduction gain. Nevertheless, the different output voltage level does not specify the performance of this system. In Fig. 9(b), we plot the SNR of this system as a function of analyte concentration. The graph shows that the HDL of the system, similar to the previous example, is limited by the saturation. The MDL, on the other hand, is almost constant and it seems to be independent of interference. By looking carefully at Fig. 9(b), we can see that the SNR at low analyte concentration deviates very much from the QL-SNR, which is an indication that the additive noise of the transducer is dominating the SNR at low concentration levels. To explore this hypothesis, in Fig. 9(c), we have plotted the simulation results of the same assay but with a highperformance photodetector transducer system which has an integration time of $100 \mathrm{~s}$ (ten times more transduction gain) and 100 times less dark current. As illustrated, on the HDL side of SNR for the modified system is not improved, yet the MDL is lowered drastically, making the low concentration SNR interference limited and closer to QL-SNR. The overall improvement in DR with the modified transducer is as high as $40 \mathrm{~dB}$.

TABLE III. Characteristics of the fluorescence-based biosensor.

\begin{tabular}{ll}
\hline \hline Capturing probability & $x_{E}=2.7 \times 10^{-5}$ \\
Probe density & $n_{Y}=3.2 \times 10^{6}$ \\
Reaction chamber volume & $V=10 \mu \mathrm{l}$ \\
Photon flux transfer function & $\Delta I_{\text {flux }}=100$ photons/target s \\
Transduction matrix & $T=0.02 \times \mu \mathrm{V} /$ target \\
Transducer noise & $\sigma_{T}=10 \mu \mathrm{V}+2 \times 10^{-8} \sqrt{\operatorname{targets}}$ \\
Interferer capturing probability & $x_{E}^{(1)}=2.7 \times 10^{-8}$ \\
Interferer variation & $\sigma_{(1)}=0$ \\
\hline
\end{tabular}
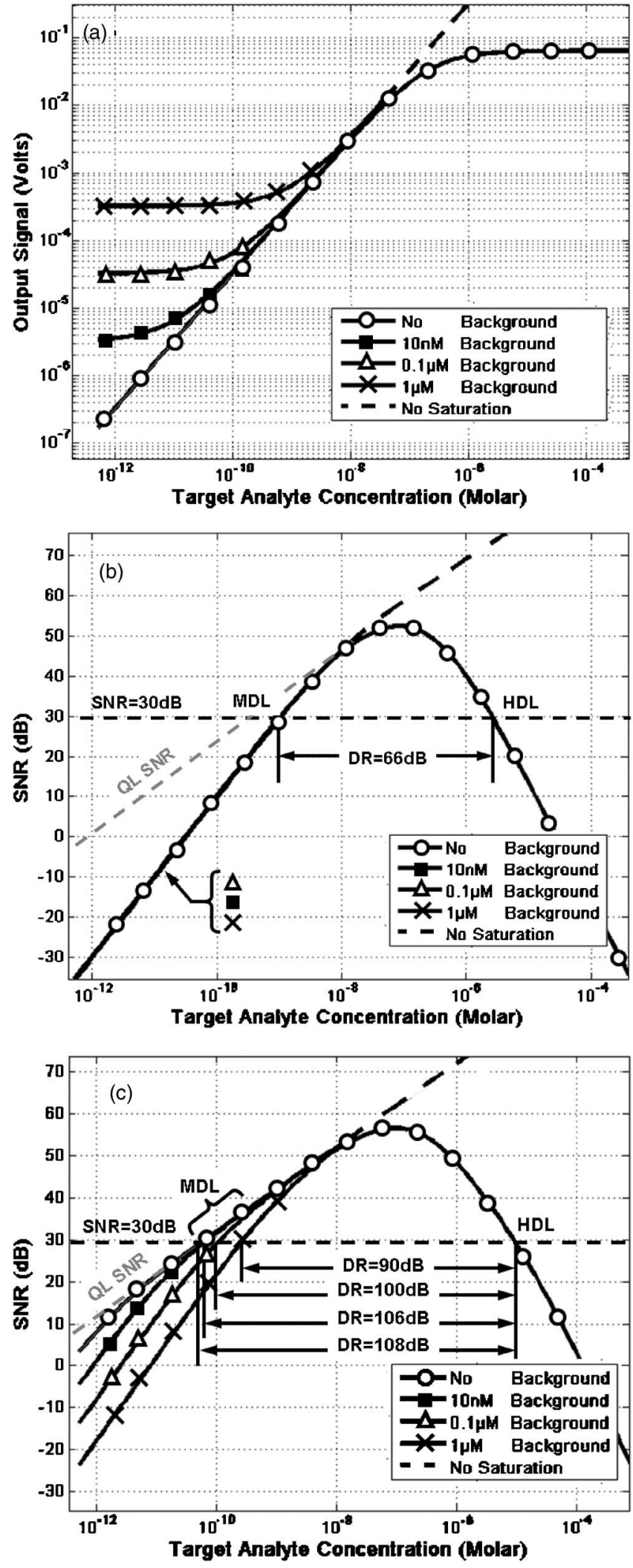

FIG. 9. Simulation result of fluorescence-based biosensor. In (a) the biosensor output signal is plotted vs the target analyte concentration. In (b) the SNR of the system is plotted and the DR is shown for the $\mathrm{SNR}_{\min }$ of $30 \mathrm{~dB}$. In (c) the SNR of the system is shown where the integration time (i.e., transduction gain) is increased by one order of magnitude while the photodetector dark-current noise power is decreased ten times.

By examining Figs. 7 and 9 we can compare the performance of both. The fluorescent-based biosensor with the ini- 

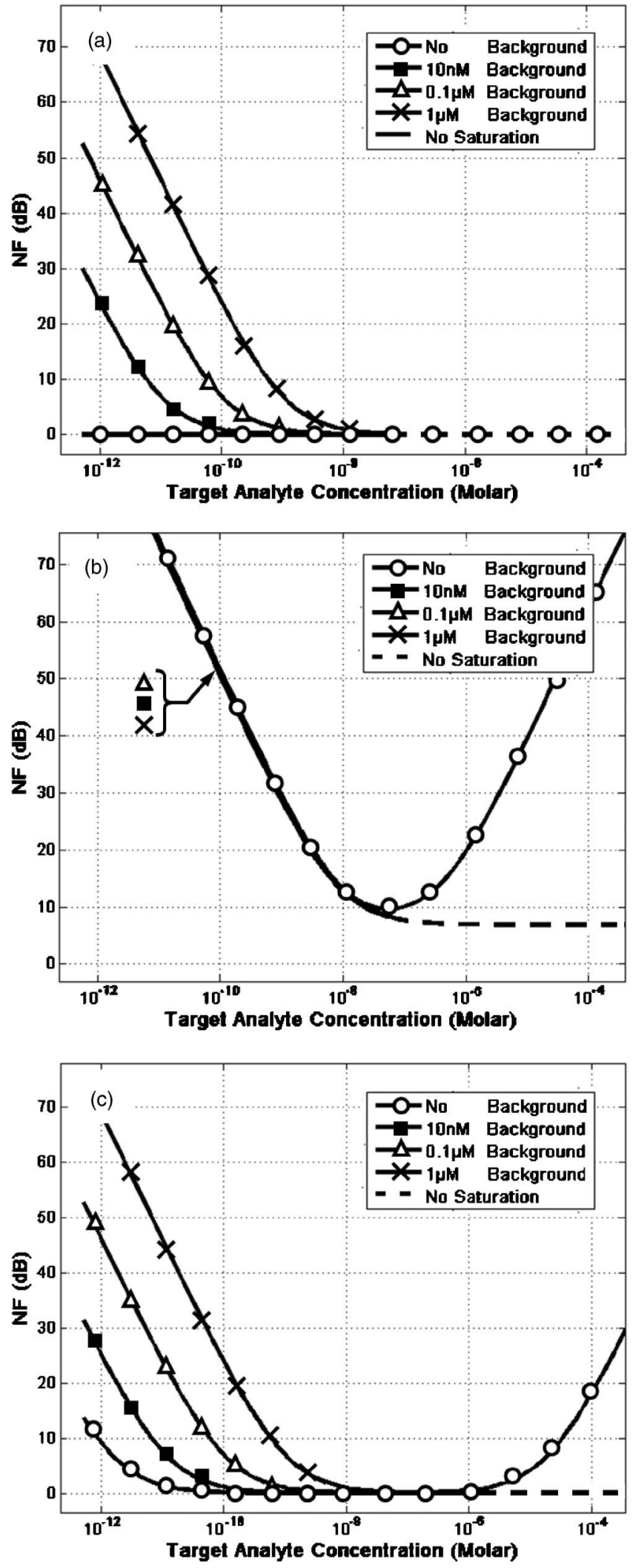

FIG. 10. Noise figure (NF) of the biosensor platforms, where (a) is for the microfabricated impedimetric biosensor, (b) is for the fluorescence-based biosensor, and (c) is for the modified (low-noise) fluorescence-based biosensor.

tial transducer had less DR compared to the impedimetric biosensor. However, with the improved transducer, the performance became comparable. This particular behavior can be better observed in the NF plots for both systems. In Fig.
10 we show the NF of the example systems, which demonstrate the fact that the transducer of the impedimetric biosensor does a better job than the fluorescent-based biosensor. However, when we modify and improve the noise performance of the photodetector, the performances of both platforms particularly in the low analyte concentration level become comparable. This is consistent with the SNR results of Figs. 7 and 9.

\section{CONCLUSION}

In this paper we present a comprehensive stochastic model describing the measurement uncertainty, output signal, and limits of detection of affinity-based biosensors. In our method, the biochemical events (motion and capturing) within the biosensor platform are modeled by a Markov stochastic process. To generalize this model for practical biosensors which have detection systems in addition to the biochemical assay, we incorporated into the model the transducer and other signal amplification stages. Using this technique, we are able to evaluate not only the output signal and the statistics of its fluctuation but also the noise contributions of each stage of the biosensor platform. Furthermore, we apply our formulations to define for biosensors the signal-to-noise ratio (SNR), noise figure (NF), and detection dynamic range (DR) as a function of target analyte concentration, background and interference molecules, and number of capturing probes.

The model presented in this paper is capable of quantifying some of the important characteristics of biosensors such as the SNR degradation due to saturation and also signal-corrupting effects of nonspecific bindings. In addition, the performance metrics derived from our model (e.g., SNR and MDL) are, in fact, independent of the biosensor platform and hence ideal comparing biosensor systems. In order to construct the models there are two fundamental parameters which one needs to calculate or empirically measure. One is the probability of analyte capturing in the biosensor system, and the other is the transducer gain.

It is important to realize that the presented noise model and the related discussions in this paper are only applicable to biosensor platforms which have reached equilibrium. Kinetic assays or biosensors which observe the dynamics of the binding processes are not included in our formulations.

${ }^{1}$ M. Schena, Microarray Analysis (Wiley, New York, 2003).

${ }^{2}$ M. Schena, Protein Microarrays (Johns and Bartlett, Sudbury, MA, 2003). ${ }^{3}$ Immunoassays, 1st ed., edited by E. P. Diamandis and T. K. Christopoulos (Academic, San Diego, 1996).

${ }^{4}$ G. T. A. Kovacs, Micromachined Transducers (McGraw-Hill, New York, 1998).

${ }^{5}$ H. C. Berg, Random Walks in Biology, (Princeton University Press, Princeton, NJ, 1993).

${ }^{6}$ N. Levine, Quantum Chemistry, 5th ed. (Prentice-Hall, New York, 1999).

${ }^{7}$ N. G. Van Kampen, Stochastic Processes in Physics and Chemistry (North-Holland, Amsterdam, 1981).

${ }^{8}$ J. I. Steinfield, J. S. Fransisco, and W. L. Hase, Chemical Kinetics and Dynamics, 2nd ed. (Prentice-Hall, New York, 1998).

${ }^{9}$ J. Hubble, Biotechnol. Lett. 22, 1483 (2000).

${ }^{10} \mathrm{~K}$. Linneti and M. Kondratovich, Clin. Chem. 50, 732 (2004).

${ }^{11}$ A. Hassibi, S. Zahedi, R. Navid, R. W. Dutton, and T. H. Lee, J. Appl. Phys. 97, 084701 (2005).

${ }^{12}$ R. Lacowicz, Principles of Fluorescence Spectroscopy, 2nd ed. (Plenum, New York, 1999). 
${ }^{13}$ A. J. Bard and L. J. Faulkner, Electrochemical Methods: Fundamentals and Applications, 2nd ed., (Wiley, New York, 2001).

${ }^{14}$ L. C. Rogers and D. Williams, Diffusions, Markov Processes, and Martingales, 2nd ed. (Cambridge University Press, Cambridge, 2000).

${ }^{15}$ R. S. Berry, S. A. Rice, and J. Ross, Physical Chemistry, 2nd ed. (Oxford University Press, New York, 2000).

${ }^{16}$ K. Salama, H. Eltoukhy, A. Hassibi, and A. El Gamal, Biosens. Bioelectron. 19, 1377 (2004), special issue on micro and nano Bioengineering.

${ }^{17}$ H. A. Hauss, Electromagnetic Noise and Quantum Optical Measurements (Springer, New York, 2000).

${ }^{18}$ A. Van der Ziel, Noise in Solid State Devices and Circuits, 9th ed. (Wiley, New York, 1986).
${ }^{19}$ P. Bergveld, IEEE Trans. Biomed. Eng. 17, 70 (1970).

${ }^{20}$ J. Fritz, E. Cooper, S. Gaudet, P. K. Sorger, and S. Manalis, Proc. Natl. Acad. Sci. U.S.A. 99, 14142 (2002)

${ }^{21}$ A. Hassibi, and T. H. Lee, IEEE Sens. J. 6, 1380 (2006).

${ }^{22}$ C. A. Savran and S. M. Knudson, Anal. Chem. 76, 3194 (2004).

${ }^{23}$ W. Fritzsche and T. A. Taton, Nanotechnology 14, 63 (2003).

${ }^{24}$ D. Kilian, L. D. Montgomery, W. Wang, and J. C. Tsai, Anal. Chim. Acta 444, 69 (2001).

${ }^{25}$ M. Schienle, C. Paulus, A. Frey, F. Hofmann, B. Holzapfl, P. SchindlerBauer, and R. Thewes, IEEE J. Solid-State Circuits 39, 2438 (2004).

${ }^{26}$ Y. Tu, G. Stolovitzky, and U. Klein, Proc. Natl. Acad. Sci. U.S.A. 99, 14031 (2002). 